\title{
Existence and multiplicity results for a class of degenerate quasilinear elliptic systems
}

\author{
G.A. Afrouzi* N.T. Chung $\quad$ S. Mahdavi
}

\begin{abstract}
In this paper, some existence and multiplicity results involving eigenvalues are established for a class of degenerate quasilinear elliptic system by using Ekeland's variational principle, the mountain pass theorem and the critical point theory.
\end{abstract}

\section{Introduction}

In this paper, we consider the problem

$$
\left\{\begin{array}{lc}
-\operatorname{div}\left(h_{1}(x)|\nabla u|^{p-2} \nabla u\right)=\lambda a(x)|u|^{p-2} u+F_{u}(x, u, v) & \text { in } \Omega, \\
-\operatorname{div}\left(h_{2}(x)|\nabla v|^{q-2} \nabla v\right)=\mu b(x)|v|^{q-2} v+F_{v}(x, u, v) & \text { in } \Omega, \\
u=v=0 & \text { on } \partial \Omega
\end{array}\right.
$$

where $\Omega$ is a bounded smooth domain in $\mathbb{R}^{N}, 1<p, q<N, \lambda, \mu$ are non-negative parameters, and $F \in C^{1}\left(\bar{\Omega} \times \mathbb{R}^{2}, \mathbb{R}\right)$ satisfies

$\left(F_{0}\right) F(x, 0,0)=0$ for all $x \in \bar{\Omega}$, there exist two constants $s_{0}, t_{0}>0$ such that $F_{s}(x, s, t)=F_{s}\left(x, s, t_{0}\right)$ for all $(x, s, t) \in \bar{\Omega} \times \mathbb{R} \times\left(\mathbb{R} \backslash\left[-t_{0}, t_{0}\right]\right), F_{t}(x, s, t)=$ $F_{t}\left(x, s_{0}, t\right)$ for all $(x, s, t) \in \bar{\Omega} \times\left(\mathbb{R} \backslash\left[-s_{0}, s_{0}\right]\right) \times \mathbb{R}$,

\footnotetext{
${ }^{*}$ Corresponding author

Received by the editors in May 2013 - In revised form in September 2014.

Communicated by D. Bonheure.

2010 Mathematics Subject Classification : 35J65; 35B30, 34A15.

Key words and phrases : Degenerate quasilinear elliptic systems; Existence and multiplicity results; Mountain pass theorem; Critical point theory.
} 
and the growth condition:

$$
\lim _{|s| \rightarrow \infty} \frac{F_{s}(x, s, t)}{a(x)|s|^{p-1}}=0, \quad \lim _{|t| \rightarrow \infty} \frac{F_{t}(x, s, t)}{b(x)|t|^{q-1}}=0
$$

uniformly in $(x, t) \in \bar{\Omega} \times \mathbb{R}$ and $(x, s) \in \bar{\Omega} \times \mathbb{R}$ respectively, where $\nabla F=\left(F_{s}, F_{t}\right)$ stands for the gradient of $F$ with respect to $(s, t) \in \mathbb{R}^{2}$.

We observe that there exists a vast literature on non-uniformly nonlinear elliptic problems in bounded or unbounded domains. Many authors studied the existence of solutions for such problems (equations or systems), for instance see $[5,6,7,8,9,10,14,17,18,19,21,22]$. In a recent paper Caldiroli et al. [5] considered the Dirichlet elliptic problem

$$
-\operatorname{div}(h(x) \nabla u)=\lambda u+g(x, u) \text { in } \Omega,
$$

where $\Omega$ is a (bounded or unbounded) domain in $\mathbb{R}^{N}(N \geq 2)$, and $h$ is a nonnegative measurable weighted function that is allowed to have "essential" zeroes at some points in $\Omega$, i.e., the function $h$ can have at most a finite number of zeroes in $\Omega$.

The results in [5] were used by Zographopoulos [21], Zhang et al. [18] and Chung et al. $[7,8,9]$ to study the existence of solutions for a class of degenerate elliptic systems.

In [1], Afrouzi et al. motivated by the paper of Ou and Tang [15], obtained three solutions for problem (1.1) in the case $h_{1}=h_{2} \equiv 1$ as the parameters $\lambda$ and $\mu$ approach $\lambda_{1}$ and $\mu_{1}$ from the left, respectively. Inspired by $[1,11,13,15,20]$ and [22], the goal of this paper is to prove some existence and multiplicity results involving eigenvalues for a class of degenerate elliptic systems.

Let $h_{1}, h_{2}$ be positive weight functions a.e. in $\Omega$ such that

$$
\begin{array}{ll}
h_{1} \in L_{l o c}^{1}(\Omega), & h_{1}^{-s_{1}} \in L^{1}(\Omega), \quad s_{1} \in\left(\frac{N}{p}, \infty\right) \cap\left[\frac{1}{p-1}, \infty\right), \\
h_{2} \in L_{l o c}^{1}(\Omega), & h_{2}^{-s_{2}} \in L^{1}(\Omega), \quad s_{2} \in\left(\frac{N}{q}, \infty\right) \cap\left[\frac{1}{q-1}, \infty\right) .
\end{array}
$$

We define the spaces $W_{0}^{1, p}\left(\Omega, h_{1}\right), W_{0}^{1, q}\left(\Omega, h_{2}\right)$ as being the completions of $C_{0}^{\infty}(\Omega)$ with respect to the norms defined by

$$
\begin{gathered}
\|u\|_{h_{1}, p}=\left(\int_{\Omega} h_{1}(x)|\nabla u|^{p} d x\right)^{\frac{1}{p}}, \quad \forall u \in C_{0}^{\infty}(\Omega), \\
\|v\|_{h_{2}, q}=\left(\int_{\Omega} h_{2}(x)|\nabla v|^{q} d x\right)^{\frac{1}{q}}, \quad \forall v \in C_{0}^{\infty}(\Omega)
\end{gathered}
$$

respectively, and set $H=W_{0}^{1, p}\left(\Omega, h_{1}\right) \times W_{0}^{1, q}\left(\Omega, h_{2}\right)$. It is clear that $H$ is a reflexive Banach space under the norm

$$
\|w\|_{H}=\|u\|_{h_{1}, p}+\|v\|_{h_{2}, q}
$$

for all $w=(u, v) \in H$. 
We recall some facts about the homogeneous degenerate eigenvalue problem

$$
\begin{cases}-\operatorname{div}\left(h_{1}(x)|\nabla u|^{p-2} \nabla u\right)=\lambda a(x)|u|^{p-2} u & \text { in } \Omega, \\ u=0 & \text { on } \partial \Omega,\end{cases}
$$

where $\Omega$ is a bounded domain in $\mathbb{R}^{N}, 1<p<N$ and $h_{1}$ satisfies (1.4). With the number $s$ given in (1.4) we define

$$
p_{s_{1}}=\frac{p s_{1}}{s_{1}+1}, \quad p_{s_{1}}^{*}=\frac{N p_{s_{1}}}{N-p_{s_{1}}}=\frac{N p s_{1}}{N\left(s_{1}+1\right)-p s_{1}}>p .
$$

In this paper, we assume that the coefficient function $a$ satisfies

$$
\operatorname{meas}\{x \in \Omega: a(x)>0\}>0, \quad a \in L^{\frac{r_{1}}{r_{1}-p}}(\Omega) \text {, for some } p<r_{1}<p_{s_{1}}^{*} .
$$

The authors in [13] established the existence of sequence of positive eigenvalues $\left\{\lambda_{k}\right\}_{k \in N}$ where $\lambda_{k}$ determined by the following way. Let

$$
\begin{gathered}
M_{1}=\left\{u \in W_{0}^{1, p}\left(\Omega, h_{1}\right): \quad \int_{\Omega} a(x)|u|^{p} d x=1\right\}, \\
I_{1}(u)=\int_{\Omega} h_{1}(x)|\nabla u|^{p} d x, \quad u \in W_{0}^{1, p}\left(\Omega, h_{1}\right) .
\end{gathered}
$$

Then we get

$$
\lambda_{k}=\inf _{A_{1} \in \sum_{k}} \sup _{u \in A_{1}} I_{1}(u),
$$

where $\sum_{k}=\left\{A_{1} \subset M_{1} \quad: \quad\right.$ there exists a continuous, odd and surjective $\left.\gamma_{1}: S^{k-1} \rightarrow A_{1}\right\}$ and $S^{k-1}$ denotes the unit sphere in $\mathbb{R}^{k}, \lambda_{k} \rightarrow \infty$ as $k \rightarrow \infty$. It has been proved in [11, Chapter 3] that the principal eigenvalue $\lambda_{1}$ is simple and isolated and all eigenfunctions corresponding to $\lambda_{1}$ do not change sign in $\Omega$. It is obvious that

$$
\lambda_{1}=\inf _{u \in M_{1}} I_{1}(u)
$$

which implies that

$$
\int_{\Omega} h_{1}(x)|\nabla u|^{p} d x \geq \lambda_{1} \int_{\Omega} a(x)|u|^{p} d x, \quad \forall u \in W_{0}^{1, p}\left(\Omega, h_{1}\right) .
$$

Besides, the corresponding normalized eigenfunction $\varphi_{1}$ belongs to $W_{0}^{1, p}\left(\Omega, h_{1}\right)$.

Similarly, we consider the eigenvalue problem

$$
\left\{\begin{array}{lc}
-\operatorname{div}\left(h_{2}(x)|\nabla v|^{q-2} \nabla v\right)=\mu b(x)|v|^{q-2} v & \text { in } \Omega \\
v=0 & \text { on } \partial \Omega .
\end{array}\right.
$$

Where $h_{2}$ satisfies condition (1.5), meas $\{x \in \Omega: b(x)>0\}>0, \quad b \in L^{\frac{r_{2}}{r_{2}-q}}(\Omega)$ for some $q<r_{2}<q_{s_{2}}^{*}$ where $q_{s_{2}}^{*}=\frac{N q s_{2}}{N\left(s_{2}+1\right)-q s_{2}}>q$. Let

$$
M_{2}=\left\{v \in W_{0}^{1, q}\left(\Omega, h_{2}\right): \quad \int_{\Omega} b(x)|v|^{q} d x=1\right\}
$$


and

$$
I_{2}(v)=\int_{\Omega} h_{2}(x)|\nabla v|^{q} d x, \quad v \in W_{0}^{1, q}\left(\Omega, h_{2}\right) .
$$

By a standard argument, problem (1.9) has a sequence of eigenvalues with the variational characterization

$$
\mu_{k}=\inf _{A_{2} \in \sum_{k}^{\prime}} \sup _{v \in A_{2}} I_{2}(v)
$$

where $\sum_{k}^{\prime}=\left\{A_{2} \subset M_{2}:\right.$ there exists a continuous, odd and surjective $\gamma_{2}: S^{k-1} \rightarrow$ $\left.A_{2}\right\}$ and $\mu_{k} \rightarrow \infty$ as $k \rightarrow \infty$. We also have

$$
\mu_{1}=\inf _{v \in M_{2}} I_{2}(v)
$$

which implies that

$$
\int_{\Omega} h_{2}(x)|\nabla v|^{q} d x \geq \mu_{1} \int_{\Omega} b(x)|v|^{q} d x, \quad \forall v \in W_{0}^{1, q}\left(\Omega, h_{2}\right) .
$$

Besides, the corresponding normalized eigenfunction $\psi_{1}$ belongs to $W_{0}^{1, q}\left(\Omega, h_{2}\right)$.

Let

$$
\begin{aligned}
W^{\prime}=\{w=(u, v) \in H: & \int_{\Omega} a(x)\left|\varphi_{1}\right|^{p-2} \varphi_{1} u d x=0 \\
& \text { and } \left.\int_{\Omega} b(x)\left|\psi_{1}\right|^{q-2} \psi_{1} v d x=0\right\} .
\end{aligned}
$$

We can easily prove that $W^{\prime}$ is complementary subspace of $W=\left\langle\varphi_{1}\right\rangle \times\left\langle\psi_{1}\right\rangle$. Therefore, we have the following direct sum

$$
H=W \oplus W^{\prime} .
$$

Now we are ready to state our main results.

Theorem 1.1. Suppose that the nonlinearity F satisfies the conditions $\left(F_{0}\right),(1.2)$ and

$$
\lim _{|s|,|t| \rightarrow \infty} F\left(x, s \varphi_{1}, t \psi_{1}\right)=\infty
$$

uniformly in $x \in \Omega$. Then for any $\lambda<\lambda_{1}$ and $\mu<\mu_{1}$ sufficiently close to $\lambda_{1}$ and $\mu_{1}$, problem (1.1) has at least three solutions.

Theorem 1.2. Suppose that the nonlinearity $F$ satisfies satisfies the conditions $\left(F_{0}\right),(1.2)$ and

$$
\lim _{|(s, t)| \rightarrow \infty}\left(\frac{1}{p} F_{s}(x, s, t) s+\frac{1}{q} F_{t}(x, s, t) t-F(x, s, t)\right) d x=-\infty .
$$

Then for $\lambda_{k}<\lambda<\lambda_{k+1}, \mu_{k}<\mu<\mu_{k+1}$ and also for the case $\lambda=\lambda_{k}, \mu=\mu_{k}$, problem (1.1) has at least one solution. 


\section{Preliminaries}

For each $\lambda, \mu \in \mathbb{R}$, let $I: H \rightarrow \mathbb{R}$ be the functional defined by

$$
\begin{array}{r}
I_{\lambda, \mu}(u, v)=\frac{1}{p} \int_{\Omega} h_{1}(x)|\nabla u|^{p} d x+\frac{1}{q} \int_{\Omega} h_{2}(x)|\nabla v|^{p} d x-\frac{\lambda}{p} \int_{\Omega} a(x)|u|^{p} d x \\
-\frac{\mu}{q} \int_{\Omega} b(x)|v|^{q} d x-\int_{\Omega} F(x, u, v) d x .
\end{array}
$$

Since the potential $F$ satisfies (1.2), it follows that $I_{\lambda, \mu} \in C^{1}(H, \mathbb{R})$ and its derivative is

$$
\begin{array}{r}
I_{\lambda, \mu}^{\prime}(u, v)\left(\eta_{1}, \eta_{2}\right)=\int_{\Omega} h_{1}(x)|\nabla u|^{p-2} \nabla u \cdot \nabla \eta_{1} d x+\int_{\Omega} h_{2}(x)|\nabla v|^{q-2} \nabla v \cdot \nabla \eta_{2} d x \\
-\lambda \int_{\Omega} a(x)|u|^{p-2} u \eta_{1} d x-\mu \int_{\Omega} b(x)|v|^{q-2} v \eta_{2} d x \\
\quad-\int_{\Omega} F_{u}(x, u, v) \eta_{1} d x-\int_{\Omega} F_{v}(x, u, v) \eta_{2} d x
\end{array}
$$

for all $(u, v),\left(\eta_{1}, \eta_{2}\right) \in H$. In addition, $(u, v) \in H$ is a weak solution of problem (1.1) if and only if $(u, v)$ is a critical point of $I_{\lambda, \mu}$. It is well known that the following lemma holds.

Lemma 2.1 (see [22]). Assume that $\Omega$ is a bounded domain in $\mathbb{R}^{N}$ and the weight $h$ satisfies

$$
h \in L_{l o c}^{1}(\Omega), \quad h^{-s} \in L^{1}(\Omega), \quad s \in\left(\frac{N}{p}, \infty\right) \cap\left[\frac{1}{p-1}, \infty\right) .
$$

Then the following embeddings hold true

(i) $W_{0}^{1, p}(\Omega, h) \hookrightarrow L^{p_{s}^{*}}(\Omega)$ continuously for $1<p_{s}^{*}<N$;

(ii) $W_{0}^{1, p}(\Omega, h) \hookrightarrow L^{r}(\Omega)$ compactly for $r \in\left[1, p_{s}^{*}\right)$.

Putting

$$
\begin{array}{ll}
U:=\left\{u \in W_{0}^{1, p}\left(\Omega, h_{1}\right):\right. & \left.\int_{\Omega} a(x)\left|\varphi_{1}\right|^{p-2} \varphi_{1} u d x=0\right\}, \\
V:=\left\{v \in W_{0}^{1, q}\left(\Omega, h_{2}\right):\right. & \left.\int_{\Omega} b(x)\left|\psi_{1}\right|^{q-2} \psi_{1} v d x=0\right\} .
\end{array}
$$

Then $U$ and $V$ are closed subspaces and they hold that

$$
W_{0}^{1, p}\left(\Omega, h_{1}\right)=U \oplus\left\langle\phi_{1}\right\rangle, \quad W_{0}^{1, q}\left(\Omega, h_{2}\right)=V \oplus\left\langle\psi_{1}\right\rangle .
$$

Proposition 2.2. Set

$$
\bar{\lambda}=\inf _{u \in U \backslash\{0\}} \frac{\|u\|_{h_{1}, p}^{p}}{\int_{\Omega} a(x)|u|^{p} d x}, \quad \bar{\mu}=\inf _{v \in V \backslash\{0\}} \frac{\|v\|_{h_{2}, q}^{q}}{\int_{\Omega} b(x)|v|^{q} d x} .
$$

Then we have $\lambda_{1}<\bar{\lambda}$ and $\mu_{1}<\bar{\mu}$, where $\lambda_{1}$ and $\mu_{1}$ are defined above. 
Proof. Indeed, we argue by contradiction. Since $\lambda_{1} \leq \frac{\|u\|_{h_{1}, p}^{p}}{\int_{\Omega} a(x)|u|^{p} d x}$ holds for each $u \neq 0$, we assume that $\lambda_{1}=\bar{\lambda}$, i.e.

$$
\bar{\lambda}=\inf _{u \in W_{0}^{1, p}\left(\Omega, h_{1}\right) \backslash\{0\}} \frac{\|u\|_{h_{1}, p}^{p}}{\int_{\Omega} a(x)|u|^{p} d x} .
$$

Then, we may suppose that there exist a sequence $\left\{u_{n}\right\}_{n} \subset U$ and $u \in W^{1, p}\left(\Omega, h_{1}\right)$ such that

$$
\int_{\Omega} a(x)\left|u_{n}\right|^{p} d x=1, \quad \lim _{n \rightarrow \infty}\left\|u_{n}\right\|_{h_{1}, p}^{p}=\bar{\lambda}, \quad u_{n} \rightarrow u \in W_{0}^{1, p}\left(\Omega, h_{1}\right),
$$

and thus $u_{n} \rightarrow u$ strongly in $L^{p}(\Omega)$.

Since $U$ is closed and $u_{n}$ converges strongly to $u$ in $L^{p}(\Omega)$, we get $u \in U$ and $\int_{\Omega} a(x)|u|^{p} d x=1$ holds. Using weak lower semicontinuity of the norm and the variational characterization of $\lambda_{1}$, we get

$$
\lambda_{1} \leq\|u\|_{W_{0}^{1, p}}^{p} \leq \lim _{n \rightarrow \infty} \inf \left\|u_{n}\right\|_{W_{0}^{1, p}}^{p}=\bar{\lambda}=\lambda_{1},
$$

which implies that $u= \pm \varphi_{1}$. This contradicts $\pm \varphi_{1} \notin U$. Analogously, we can prove that $\mu_{1}<\bar{\mu}$.

In what follows, we recall some basic definitions and results to prove our theorems.

Definition 2.3. The functional $I$ is said to satisfy the Palais-Smale condition at level $c,(P S)_{c}$, if every sequence for which

$$
I\left(w_{n}\right) \rightarrow c, \quad\left\|I^{\prime}\left(w_{n}\right)\right\|_{H^{*}} \rightarrow 0,
$$

possesses a convergent subsequence. When $I$ satisfies $(P S)_{c}$, for all $c \in \mathbb{R}$, we simply say that $I$ satisfies the $(P S)$ condition.

Definition 2.4. The functional $I$ satisfies $(\mathrm{Ce})_{c}$ condition at level $c \in R$ if any sequence $\left\{w_{n}\right\} \subset H$ such that

$$
I\left(w_{n}\right) \rightarrow c, \quad\left(1+\left\|w_{n}\right\|_{H}\right)\left\|I^{\prime}\left(w_{n}\right)\right\|_{H^{*}} \rightarrow 0 \text { as } n \rightarrow \infty,
$$

has a convergent subsequence. The functional $I$ satisfies $(\mathrm{Ce})$ condition if $I$ satisfies $(\mathrm{Ce})_{c}$ at any $c \in R$. The $(\mathrm{Ce})$ condition was introduced by Cerami, it is a weaker version of the $(P S)$ condition (see [4]).

Definition 2.5 (see [16]). Let $Q, Q_{0}$ be submanifolds of a Banach space $X$ with $Q_{0} \subset Q, S$ be a closed subset of a Banach space $Y$ and $\Gamma \subset C^{0}\left(Q_{0}, Y \backslash S\right)$. We say $S$ and $\left(Q, Q_{0}\right)$ are $\Gamma-$ linking if for any map $h \in C^{0}(Q, Y)$ such that $\left.h\right|_{Q_{0}} \in \Gamma$ there holds $h(Q) \cap S \neq \varnothing$.

Lemma 2.6 (see [20]). Suppose that $X, Y$ are Banach spaces. Consider submanifolds $Q, Q_{0} \subset X$ with $Q_{0} \subset Q$ and a closed subset $S \subset Y$ such that $\left(Q, Q_{0}\right)$ and $S$ are $\Gamma$-linking. Let $\Gamma^{*}=\left\{\gamma \in C^{0}(Q, Y):\left.\gamma\right|_{Q_{0}} \in \Gamma\right\}$. For $f \in C^{1}(Y, R)$ satisfies 
(1) $\exists \gamma_{0} \in \Gamma^{*}$ such that $\sup _{x \in Q} f\left(\gamma_{0}(x)\right)<+\infty$;

(2) $\exists \beta>\alpha$ such that $\inf _{y \in S} f(y) \geq \beta$ and $\sup _{x \in Q_{0}} f(\gamma(x)) \leq \alpha$ for $\gamma \in \Gamma^{*}$;

(3) $f$ satisfies the $(\mathrm{Ce})$ condition.

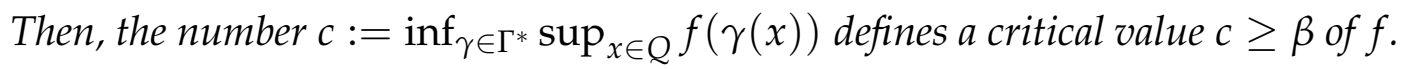

For the proof of Lemma 2.6 we refer the readers to [20].

\section{Proofs of the main results}

\subsection{Proof of Theorem 1.1.}

We will prove Theorem 1.1 by using Ekeland's variational principal and the mountain pass theorem. More precisely, the proof will be divided into four steps.

Step 1. For $\lambda<\lambda_{1}$ and $\mu<\mu_{1}$, the functional $I_{\lambda, \mu}$ is coercive in $H, I_{\lambda, \mu}$ is bounded from below on $W^{\prime}$ and there is a constant $m$, independent of $\lambda$, $\mu$, such that $\inf _{W^{\prime}} I_{\lambda, \mu} \geq m$.

From the conditions $\left(F_{0}\right),(1.2)$ and the continuity of the potential $F$, for any $\varepsilon>0$, there exists a positive constant $M_{\mathcal{\varepsilon}}=M(\varepsilon)$ such that

$$
\left|\frac{\partial F}{\partial s}(x, s, t)\right| \leq \varepsilon a(x)|s|^{p-1}+M_{\epsilon}, \quad\left|\frac{\partial F}{\partial t}(x, s, t)\right| \leq \varepsilon b(x)|t|^{q-1}+M_{\epsilon}
$$

for all $(x, s, t) \in \bar{\Omega} \times \mathbb{R}^{2}$. By Hölder's inequality, we have

$$
\begin{aligned}
F(x, u, v) & =\int_{0}^{u} \frac{\partial F}{\partial s}(x, s, v) d s+F(x, 0, v) \\
& =\int_{0}^{u} \frac{\partial F}{\partial s}(x, s, v) d s+\int_{0}^{v} \frac{\partial F}{\partial s}(x, 0, s) d s+F(x, 0,0) \\
& \leq \int_{0}^{u}\left(\varepsilon a(x)|s|^{p-1}+M_{\mathcal{\varepsilon}}\right) d s+\int_{0}^{v}\left(\varepsilon b(x)|s|^{q-1}+M_{\mathcal{\varepsilon}}\right) d s \\
& =\frac{\varepsilon}{p} a(x)|u|^{p}+M_{\varepsilon} u+\frac{\varepsilon}{q} b(x)|v|^{q}+M_{\mathcal{\varepsilon}} v
\end{aligned}
$$

for all $(u, v) \in H$. Hence,

$$
\begin{aligned}
& \left|\int_{\Omega} F(x, u, v) d x\right| \leq \int_{\Omega}|F(x, u, v)| d x \\
& \leq \epsilon\left(\frac{1}{p} \int_{\Omega} a(x)|u|^{p} d x+\frac{1}{q} \int_{\Omega} b(x)|v|^{q} d x\right)+M_{\epsilon} \int_{\Omega} u d x+M_{\epsilon} \int_{\Omega} v d x \\
& \leq \frac{\epsilon}{p \lambda_{1}} \int_{\Omega} h_{1}(x)|\nabla u|^{p} d x+\frac{\epsilon}{q \mu_{1}} \int_{\Omega} h_{2}(x)|\nabla v|^{q} d x \\
& \quad+M_{\mathcal{\varepsilon}}|\Omega|^{\frac{p-1}{p}} S_{1}\left(\int_{\Omega} h_{1}(x)|\nabla u|^{p} d x\right)^{\frac{1}{p}}+M_{\mathcal{\varepsilon}}|\Omega|^{\frac{q-1}{q}} S_{2}\left(\int_{\Omega} h_{2}(x)|\nabla v|^{q} d x\right)^{\frac{1}{q}}
\end{aligned}
$$




$$
\begin{aligned}
& \leq \frac{\varepsilon}{p \lambda_{1}} \int_{\Omega} h_{1}(x)|\nabla u|^{p} d x+\frac{\varepsilon}{q \mu_{1}} \int_{\Omega} h_{2}(x)|\nabla v|^{q} d x \\
& \quad+C_{1}\left[\left(\int_{\Omega} h_{1}(x)|\nabla u|^{p} d x\right)^{\frac{1}{p}}+\left(\int_{\Omega} h_{2}(x)|\nabla v|^{q} d x\right)^{\frac{1}{q}}\right],
\end{aligned}
$$

where $S_{1}, S_{2}$ are the embedding constants of $W_{0}^{1, p}\left(\Omega, h_{1}\right) \hookrightarrow L^{p}(\Omega), W_{0}^{1, q}\left(\Omega, h_{2}\right) \hookrightarrow$ $L^{q}(\Omega)$, respectively and $C_{1}=\max \left\{M_{\mathcal{\varepsilon}}|\Omega|^{\frac{p-1}{p}} S_{1}, M_{\varepsilon}|\Omega|^{\frac{q-1}{q}} S_{2}\right\}$.

For $\lambda<\lambda_{1}$ and $\mu<\mu_{1}$, from the definition of $\lambda_{1}, \mu_{1}$ and (3.1), we get

$$
\begin{aligned}
I_{\lambda, \mu}(u, v)=\frac{1}{p} \int_{\Omega} h_{1}(x)|\nabla u|^{p} d x+\frac{1}{q} \int_{\Omega} h_{2}(x)|\nabla v|^{q} d x-\frac{\lambda}{p} \int_{\Omega} a(x)|u|^{p} d x \\
\quad-\frac{\mu}{q} \int_{\Omega} b(x)|v|^{q} d x-\int_{\Omega} F(x, u, v) d x \\
\geq \frac{1}{p}\left(1-\frac{\lambda}{\lambda_{1}}-\frac{\varepsilon}{\lambda_{1}}\right) \int_{\Omega} h_{1}(x)|\nabla u|^{p} d x \\
\quad+\frac{1}{q}\left(1-\frac{\mu}{\mu_{1}}-\frac{\epsilon}{\mu_{1}}\right) \int_{\Omega} h_{2}(x)|\nabla v|^{q} d x-C_{1}\|(u, v)\|_{H} .
\end{aligned}
$$

Letting $\varepsilon=\min \left\{\frac{\lambda_{1}-\lambda}{2}, \frac{\mu_{1}-\mu}{2}\right\}$, it follows that $I_{\lambda, \mu}$ is coercive in $H$.

Similarly, from Proposition 2.2 we obtain

$$
\begin{aligned}
I_{\lambda, \mu}(u, v) \geq \frac{1}{p} & \left(1-\frac{\lambda}{\bar{\lambda}}-\frac{\varepsilon}{\lambda_{1}}\right) \int_{\Omega} h_{1}(x)|\nabla u|^{p} d x \\
& +\frac{1}{q}\left(1-\frac{\mu}{\bar{\mu}}-\frac{\varepsilon}{\mu_{1}}\right) \int_{\Omega} h_{2}(x)|\nabla v|^{q} d x-C_{1}\|(u, v)\|_{H} \\
\geq \frac{1}{p} & \left(1-\frac{\lambda_{1}}{\bar{\lambda}}-\frac{\varepsilon}{\lambda_{1}}\right) \int_{\Omega} h_{1}(x)|\nabla u|^{p} d x \\
& +\frac{1}{q}\left(1-\frac{\mu_{1}}{\bar{\mu}}-\frac{\varepsilon}{\mu_{1}}\right) \int_{\Omega} h_{2}(x)|\nabla v|^{q} d x-C_{1}\|(u, v)\|_{H}
\end{aligned}
$$

Let $\varepsilon=\frac{1}{2} \min \left\{\lambda_{1}\left(1-\frac{\lambda_{1}}{\bar{\lambda}}\right), \mu_{1}\left(1-\frac{\mu_{1}}{\bar{\mu}}\right)\right\}$. Hence $I_{\lambda, \mu}$ is coercive in $W$ and $I_{\lambda, \mu}$ is bounded from below on $W^{\prime}$, and moreover, there is a constant $m$, independent of $\lambda, \mu$, such that $\inf _{W^{\prime}} I_{\lambda, \mu} \geq m$.

Step 2. If $\lambda<\lambda_{1}$ and $\mu<\mu_{1}$ are sufficiently close to $\lambda_{1}, \mu_{1}$, we have $t_{1}^{-}<0<$ $t_{1}^{+}, t_{2}^{-}<0<t_{2}^{+}$such that $I_{\lambda, \mu}\left(t_{1}^{ \pm} \varphi_{1}, t_{2}^{ \pm} \psi_{1}\right)<m$. 
For $\lambda<\lambda_{1}$ and $\mu<\mu_{1}$, we have

$$
\begin{aligned}
I_{\lambda, \mu}\left(t_{1}^{+} \varphi_{1}, t_{2}^{+} \psi_{1}\right)=\frac{t_{1}^{+p}}{p} \int_{\Omega} h_{1}(x)\left|\nabla \varphi_{1}\right|^{p} d x+\frac{t_{2}^{+q}}{q} \int_{\Omega} h_{2}(x)\left|\nabla \psi_{1}\right|^{q} d x \\
\quad-\lambda \frac{t_{1}^{+p}}{p} \int_{\Omega} a(x)\left|\varphi_{1}\right|^{p} d x \\
\quad-\mu \frac{t_{2}^{+p}}{q} \int_{\Omega} b(x)\left|\psi_{1}\right|^{q} d x-\int_{\Omega} F\left(x, t_{1}^{+} \varphi_{1}, t_{2}^{+} \psi_{1}\right) d x \\
=\frac{t_{1}^{+p}}{p} \int_{\Omega} h_{1}(x)\left|\nabla \varphi_{1}\right|^{p} d x+\frac{t_{2}^{+q}}{q} \int_{\Omega} h_{2}(x)\left|\nabla \psi_{1}\right|^{q} d x \\
\quad-\frac{\lambda}{p \lambda_{1}} t_{1}^{+p} \int_{\Omega} h_{1}(x)\left|\nabla \varphi_{1}\right|^{p} d x \\
\quad-\frac{\mu t_{2}^{+q}}{q \mu_{1}} \int_{\Omega} h_{2}(x)\left|\nabla \psi_{1}\right|^{q} d x-\int_{\Omega} F\left(x, t_{1}^{+} \varphi_{1}, t_{2}^{+} \psi_{1}\right) d x .
\end{aligned}
$$

From Fatou's Lemma and condition (1.12), we get

$$
\int_{\Omega} F\left(x, t_{1}^{+} \varphi_{1}, t_{2}^{+} \psi_{1}\right) d x>-m+1 \text {. }
$$

For $\lambda_{1}-\frac{p \lambda_{1}}{2 t_{1}^{p}}<\lambda<\lambda_{1}$ and $\mu_{1}-\frac{q \mu_{1}}{2 t_{2}^{q}}<\mu<\mu_{1}$, combining (3.2) and (3.3) yields $I_{\lambda, \mu}\left(t_{1}^{+} \varphi_{1}, t_{2}^{+} \psi_{1}\right)<m$. A similar condition holds for $t_{1}^{-}, t_{2}^{-}<0$.

Step 3. If $\lambda<\lambda_{1}$ and $\mu<\mu_{1}$ the functional $I_{\lambda, \mu}$ satisfies the (PS) condition.

If $\left\{w_{n}\right\}=\left\{\left(u_{n}, v_{n}\right)\right\}$ is a (PS) sequence of $I_{\lambda, \mu},\left\{\left(u_{n}, v_{n}\right)\right\}$ must be bounded. Then passing to a subsequence if necessary, there exists $w=(u, v) \in H$ such that

$$
\begin{aligned}
& \left(u_{n}, v_{n}\right) \rightarrow(u, v) \text { weakly in } H, \\
& \left(u_{n}, v_{n}\right) \rightarrow(u, v) \text { strongly in } L^{p}(\Omega) \times L^{q}(\Omega) .
\end{aligned}
$$

So there exists a strictly decreasing subsequence $\varepsilon_{n}, \lim _{n \rightarrow \infty} \varepsilon_{n}=0$ such that

$$
\left|I_{\lambda, \mu}^{\prime}\left(u_{n}, v_{n}\right)\left(u_{n}-u, 0\right)\right| \leq \varepsilon_{n}\left\|\left(u_{n}-u, 0\right)\right\|_{H} .
$$

In particular,

$$
\begin{aligned}
\left.\left|\int_{\Omega} h_{1}(x)\right| \nabla u_{n}\right|^{p-2} \nabla u_{n} \nabla\left(u_{n}-u\right) d x-\lambda \int_{\Omega} a(x)\left|u_{n}\right|^{p-2} u_{n}\left(u_{n}-u\right) d x \\
-\int_{\Omega} F_{u}\left(x, u_{n}, v_{n}\right)\left(u_{n}-u\right) d x \mid \leq \varepsilon_{n}\left\|\left(u_{n}-u, 0\right)\right\|_{H} .
\end{aligned}
$$

Since $u_{n} \rightarrow u$ in $L^{p}(\Omega)$ and $v_{n} \rightarrow v$ in $L^{q}(\Omega)$, we have

$$
\int_{\Omega} a(x)\left|u_{n}\right|^{p-2} u_{n}\left(u_{n}-u\right) d x \leq\|a\|_{\frac{r_{1}}{r_{1}-p}}\left(\int_{\Omega}\left|u_{n}\right|^{r_{1}} d x\right)^{\frac{p-1}{r_{1}}}\left(\int_{\Omega}\left|u_{n}-u\right|^{r_{1}} d x\right)^{\frac{1}{r_{1}}}
$$

which approaches 0 as $n \rightarrow \infty$. 
Since the potential $F$ satisfies (1.2) we have

$$
\begin{aligned}
& \mid \int_{\Omega} F_{u}\left(x, u_{n}, v_{n}\right)\left(u_{n}-u\right) d x|\leq| \int_{\Omega}\left(\varepsilon a(x)\left|u_{n}\right|^{p-1}+M_{\varepsilon}\right)\left(u_{n}-u\right) d x \mid \\
& \leq \varepsilon\|a\|_{\frac{r_{1}}{r_{1}-p}}\left\|u_{n}\right\|_{r_{1}}^{p-1}\left\|u_{n}-u\right\|_{r_{1}} d x+M_{\mathcal{\varepsilon}}|\Omega|^{\frac{p-1}{p}}\left\|u_{n}-u\right\|_{p}
\end{aligned}
$$

which approaches 0 as $n \rightarrow \infty$.

Combining (3.4) with (3.5) and (3.6) we get

$$
\lim _{n \rightarrow \infty} \int_{\Omega} h_{1}(x)\left|\nabla u_{n}\right|^{p-2} \nabla u_{n}\left(\nabla u_{n}-\nabla u\right) d x=0 .
$$

Subtracting

$$
\int_{\Omega} h_{1}(x)|\nabla u|^{p-2} \nabla u\left(\nabla u_{n}-\nabla u\right) d x
$$

which converges to zero as $n$ tends to infinity, we conclude that

$$
\lim _{n \rightarrow \infty} \int_{\Omega} h_{1}(x)\left(\left|\nabla u_{n}\right|^{p-2} \nabla u_{n}-|\nabla u|^{p-2} \nabla u\right)\left(\nabla u_{n}-\nabla u\right) d x=0 .
$$

Next, we recall the following useful inequalities:

$$
\begin{cases}\left(|\xi|^{p-2} \xi-|\eta|^{p-2} \eta, \xi-\eta\right) \geq C_{2}(|\xi|+|\eta|)^{p-2}|\xi-\eta|^{2}, & \text { if } 1<p<2 \\ \left(|\xi|^{p-2} \xi-|\eta|^{p-2} \eta, \xi-\eta\right) \geq C_{2}|\xi-\eta|^{p}, & \text { if } p \geq 2\end{cases}
$$

for all $\xi, \eta \in \mathbb{R}^{N}$, where $C_{2}$ is a positive constant and $(.,$.$) denotes the usual$ product in $\mathbb{R}^{N}$.

As in [2], if $1<p<2$, by Hölder's inequality and substituting $z_{n}=h_{1}^{\frac{1}{p}} u_{n}$, $z=h_{1}^{\frac{1}{p}} u$ in system (3.8), there exists $C_{3}, C_{4}>0$ such that

$$
\begin{aligned}
0 & \leq \int_{\Omega}\left|\nabla z_{n}-\nabla z\right|^{p} d x \\
& =\int_{\Omega}\left|\nabla z_{n}-\nabla z\right|^{p}\left(\left|\nabla z_{n}\right|+|\nabla z|\right)^{\frac{p(p-2)}{2}}\left(\left|\nabla z_{n}\right|+|\nabla z|\right)^{\frac{p(2-p)}{2}} d x \\
& \leq\left(\int_{\Omega}\left|\nabla z_{n}-\nabla z\right|^{2}\left(\left|\nabla z_{n}\right|+|\nabla z|\right)^{p-2} d x\right)^{\frac{p}{2}}\left(\int_{\Omega}\left(\left|\nabla z_{n}\right|+|\nabla z|\right)^{p} d x\right)^{\frac{2-p}{2}} \\
& \leq C_{3}\left(\int_{\Omega}\left(\left|\nabla z_{n}\right|^{p-2} \nabla z_{n}-|\nabla z|^{p-2} \nabla z,\left(\nabla z_{n}-\nabla z\right) d x\right)^{\frac{p}{2}} \quad\left(\int_{\Omega}\left(\left|\nabla z_{n}\right|+|\nabla z|\right)^{p} d x\right)^{\frac{2-p}{2}}\right. \\
& \leq C_{4}\left(\int_{\Omega}\left(\left|\nabla z_{n}\right|^{p-2} \nabla z_{n}-|\nabla z|^{p-2} \nabla z,\left(\nabla z_{n}-\nabla z\right) d x\right)^{\frac{p}{2}}\right.
\end{aligned}
$$

which implies $\left\|u_{n}-u\right\|_{h_{1}, p} \rightarrow 0$ as $n \rightarrow \infty$. 
If $p \geq 2$, by (3.8), there exists $C_{5}>0$ such that

$$
0 \leq\left\|u_{n}-u\right\|_{h_{1}, p} \leq C_{5}\left(\int_{\Omega}\left(\left|\nabla z_{n}\right|^{p-2} \nabla z_{n}-|\nabla z|^{p-2} \nabla z,\left(\nabla z_{n}-\nabla z\right) d x\right),\right.
$$

so we get $\left\|u_{n}-u\right\|_{h_{1}, p} \rightarrow 0$ as $n \rightarrow \infty$. Therefore, $\left\|u_{n}-u\right\|_{h_{1}, p} \rightarrow 0$ for $p>1$ as $n \rightarrow \infty$, that is, $u_{n} \rightarrow u$ in $W_{0}^{1, p}\left(\Omega, h_{1}\right)$ as $n \rightarrow \infty$. Similarly, we obtain $v_{n} \rightarrow v$ in $W_{0}^{1, q}\left(\Omega, h_{2}\right)$ as $n \rightarrow \infty$. Consequently, the functional $I_{\lambda, \mu}$ satisfies the (PS) condition for all $\lambda<\lambda_{1}, \mu<\mu_{1}$.

In addition, let

$$
\sum_{ \pm}=\left\{w \in H: w= \pm\left(t_{1} \varphi_{1}, t_{2} \psi_{1}\right)+w^{\prime} \text { with } t_{1}, t_{2}>0 \text { and } w^{\prime} \in W^{\prime}\right\} .
$$

Then $I_{\lambda, \mu}$ satisfies $(P S)_{c, \Sigma_{+}}$and $(P S)_{c, \Sigma_{-}}$for all $c<m$.

Let $\left\{w_{n}\right\} \subset \sum_{+}$satisfies $I_{\lambda, \mu}\left(w_{n}\right) \rightarrow c<m$ and $I_{\lambda, \mu}^{\prime}\left(w_{n}\right) \rightarrow 0$ an $n \rightarrow \infty$. Since $I_{\lambda, \mu}$ is coercive and the potential $F$ satisfies (2), there is $w \in H$ such that $\left\|w_{n}\right\|_{H} \rightarrow\|w\|_{H}$ strongly in $H$. If $w \in \partial \sum_{+}=W^{\prime}$, since $\inf _{W^{\prime}} I_{\lambda, \mu} \geq m$, we get $I_{\lambda, \mu}\left(w_{n}\right) \rightarrow c \geq m$, which is impossible. Hence $w \in \sum_{+}$and $I_{\lambda, \mu}$ satisfies the $(P S)_{c, \Sigma_{+}}$condition. Similarly we have that $(P S)_{c, \Sigma_{-}}$holds for all $c<m$.

Step 4. If $\lambda<\lambda_{1}$ is sufficiently close to $\lambda_{1}$ and $\mu<\mu_{1}$ is sufficiently close to $\mu_{1}$, we get

$$
-\infty<\inf _{\sum_{ \pm}} I_{\lambda, \mu}<m
$$

which implies that $I_{\lambda, \mu}$ is bounded below in $\sum_{+}$. Consequently, from Ekeland's variational principle, there exists $\left\{w_{n}\right\} \subset \sum_{+}$such that $I_{\lambda, \mu}\left(w_{n}\right) \rightarrow \inf _{\sum_{+}} I_{\lambda, \mu}$ and $I_{\lambda, \mu}^{\prime}\left(w_{n}\right) \rightarrow 0$ as $n \rightarrow \infty$. Since $I_{\lambda, \mu}$ satisfies $(P S)_{c, \Sigma_{+}}$for all $c<m$, there is $z^{+} \in \sum_{+}$such that $I_{\lambda, \mu}\left(w^{+}\right)=\inf _{\sum_{+}} I_{\lambda, \mu}$, that is, the infimum is attained in $\sum_{+}$. A similar conclusion holds in $\sum_{-}$. So $I_{\lambda, \mu}$ has two distinct critical points, denoted by $w^{+}, w^{-}$. As in [15], we can obtain the third critical point $z$ of $I_{\lambda, \mu}$ by applying mountain pass theorem such that $I_{\lambda, \mu}(w)=c \geq m$.

\subsection{Proof of Theorem 1.2.}

We will verify the functional $I_{\lambda, \mu}$ satisfying the condition of Lemma 2.6. We first verify the Cerami condition.

Let $\left\{w_{n}\right\}=\left\{\left(u_{n}, v_{n}\right)\right\} \subset H$ be a $(C e)$ sequence. we first prove that $\left\{w_{n}\right\}$ is bounded in $H$, then by a standard argument, $\left\{w_{n}\right\}$ has a convergent subsequence. Supposing by contradiction that $\left\|w_{n}\right\|_{H} \rightarrow \infty$, and defining $\widehat{u}_{n}=\frac{u_{n}}{\left\|w_{n}\right\|_{H}}$, $\widehat{v}_{n}=\frac{v_{n}}{\left\|w_{n}\right\|_{H}}$, then $\widehat{w}_{n}=\left(\widehat{u}_{n}, \widehat{v}_{n}\right)$ is bounded in $H$ and

$$
\left\|\widehat{u}_{n}\right\|_{h_{1}, p}+\left\|\widehat{v}_{n}\right\|_{h_{2}, q}=1
$$

for every $n \in \mathbb{N}$. Going if necessary to a subsequence, also denoted by $\left\{\left(\widehat{u}_{n}, \widehat{v}_{n}\right)\right\}$, there is $\widehat{w}=(\widehat{u}, \widehat{v}) \in H$ such that

$$
\begin{aligned}
& \left(\widehat{u}_{n}, \widehat{v}_{n}\right) \rightarrow(\widehat{u}, \widehat{v}) \text { weakly in } H, \\
& \left(\widehat{u}_{n}, \widehat{v}_{n}\right) \rightarrow(\widehat{u}, \widehat{v}) \text { strongly in } L^{p}(\Omega) \times L^{q}(\Omega) .
\end{aligned}
$$


We have

$$
\begin{aligned}
\left|\left\langle I_{\lambda, \mu}^{\prime}\left(\widehat{u}_{n}, \widehat{v}_{n}\right),\left(\widehat{u}_{n}-\widehat{u}, 0\right)\right\rangle\right| & \leq\left\|I_{\lambda, \mu}^{\prime}\left(\widehat{u}_{n}, \widehat{v}_{n}\right)\right\|_{H^{*}}\left\|\widehat{u}_{n}-\widehat{u}\right\|_{h_{1}, p} \\
& \leq\left\|I_{\lambda}^{\prime}\left(\widehat{u}_{n}, \widehat{v}_{n}\right)\right\|_{H^{*}}\left(\left\|\widehat{u}_{n}\right\|_{h_{1}, p}+\|\widehat{u}\|_{h_{1}, p}\right)
\end{aligned}
$$

which approaches 0 as $n \rightarrow \infty$. Since $\widehat{u}_{n} \rightarrow \widehat{u}$ in $L^{p}(\Omega), \widehat{v}_{n} \rightarrow \widehat{v}$ in $\left.L^{q} \Omega\right)$, we have

$$
\lim _{n \rightarrow \infty} \int_{\Omega} a(x)\left|\widehat{u}_{n}\right|^{p-2} \widehat{u}_{n}\left(\widehat{u}_{n}-\widehat{u}\right) d x=0
$$

and

$$
\lim _{n \rightarrow \infty} \int_{\Omega} F_{u}\left(x, \widehat{u}_{n}, \widehat{v}_{n}\right)\left(\widehat{u}_{n}-\widehat{u}\right) d x=0
$$

By using

$$
\begin{aligned}
& \left\langle I_{\lambda, \mu}^{\prime}\left(\widehat{u}_{n}, \widehat{v}_{n}\right),\left(\widehat{u}_{n}-\widehat{u}, 0\right)\right\rangle=\int_{\Omega} h_{1}(x)\left|\nabla \widehat{u}_{n}\right|^{p-2} \nabla \widehat{u}_{n} \nabla\left(\widehat{u}_{n}-\widehat{u}\right) d x \\
& \quad-\lambda \int_{\Omega} a(x)\left|\widehat{u}_{n}\right|^{p-2} \widehat{u}_{n}\left(\widehat{u}_{n}-\widehat{u}\right) d x-\int_{\Omega} F_{u}\left(x, \widehat{u}_{n}, \widehat{v}_{n}\right)\left(\widehat{u}_{n}-\widehat{u}\right) d x
\end{aligned}
$$

and relations (3.9), (3.10) and (3.11), we obtain

$$
\lim _{n \rightarrow \infty} \int h_{1}(x)\left(\left|\nabla \widehat{u}_{n}\right|^{p-2} \nabla u_{n}-|\nabla \widehat{u}|^{p-2} \nabla \widehat{u}\right)\left(\nabla \widehat{u}_{n}-\nabla \widehat{u}\right) d x=0
$$

Substituting $\xi=h_{1}^{\frac{1}{p}} \widehat{u}_{n}, \eta=h_{1}^{\frac{1}{p}} \widehat{u}$ in system (3.8), we get $\left\|\widehat{u}_{n}-\widehat{u}\right\|_{h_{1}, p} \rightarrow 0$ as $n \rightarrow \infty$, then $\left\|\widehat{u}_{n}\right\|_{h_{1}, p} \rightarrow\|\widehat{u}\|_{h_{1}, p}$ as $n \rightarrow \infty$. In a similar way, we get $\left\|\widehat{v}_{n}\right\|_{h_{2}, q} \rightarrow$ $\|\widehat{v}\|_{h_{2}, q}$ as $n \rightarrow \infty$. It follows that $\|\widehat{u}\|_{h_{1}, p}+\|\widehat{v}\|_{h_{2}, q}=1$. Hence $(\widehat{u}, \widehat{v}) \neq(0,0)$. Consequently, we conclude that $\left|\left(u_{n}(x), v_{n}(x)\right)\right| \rightarrow \infty$ as $n \rightarrow \infty$ for a.e. $x \in \Omega$. On the other hand, we have

$$
\begin{aligned}
& I_{\lambda, \mu}\left(u_{n}, v_{n}\right)-I_{\lambda, \mu}^{\prime}\left(\frac{u_{n}}{p}, \frac{v_{n}}{q}\right)= \\
& \quad \int_{\Omega}\left(\frac{1}{p} F_{u}\left(x, u_{n}, v_{n}\right) u_{n}+\frac{1}{q} F_{v}\left(x, u_{n}, v_{n}\right) v_{n}-F\left(x, u_{n}, v_{n}\right)\right) d x \rightarrow c
\end{aligned}
$$

as $n \rightarrow \infty$, which contradicts (1.13). Hence, $\left\{\left(u_{n}, v_{n}\right)\right\}$ is bounded in $H$. By a standard argument, $\left\{\left(u_{n}, v_{n}\right)\right\}$ has a convergent subsequence.

Now, we are in the position to prove Theorem 1.2. We will split the proof into two cases according to the positions of $\lambda, \mu$.

Case 1: $\lambda_{k}<\lambda<\lambda_{k+1}, \mu_{k}<\mu<\mu_{k+1}$. In this case we will apply Lemma 2.6 to get solutions of problem (1.1). It follows from definition of $\lambda_{k}$ and $\mu_{k}$ there exist $A_{1} \in \sum_{k}$ and $A_{2} \in \sum_{k}^{\prime}$ such that $\sup _{u \in A_{1}} I_{1}(u)=m_{1} \in\left(\lambda_{k}, \lambda\right)$ and $\sup _{v \in A_{2}} I_{2}(v)=m_{2} \in\left(\mu_{k}, \mu\right)$ respectively.

From (3.1) and the definition of the functional $I_{\lambda, \mu}$, we deduce for any 
$(u, v) \in A_{1} \times A_{2}$ and $t>0$ that

$$
\begin{aligned}
& I_{\lambda, \mu}\left(t^{\frac{1}{p}} u, t^{\frac{1}{q}} v\right) \leq \frac{t}{p} \int_{\Omega} h_{1}(x)|\nabla u|^{p} d x+\frac{t}{q} \int_{\Omega} h_{2}(x)|\nabla v|^{q} d x-\frac{t \lambda}{p} \int_{\Omega} a(x)|u|^{p} d x \\
& \quad-\frac{t \mu}{q} \int_{\Omega} b(x)|v|^{q} d x+\left|\int_{\Omega} F\left(x, t^{\frac{1}{p}} u, t^{\frac{1}{q}} v\right) d x\right| \\
& \leq \frac{t}{p}\left(m_{1}-\lambda\right)+\frac{t}{q}\left(m_{2}-\mu\right)+t\left(\frac{\varepsilon}{p \lambda_{1}} m_{1}+\frac{\varepsilon}{q \mu_{1}} m_{2}\right) \\
& \quad+C_{1} t^{\frac{1}{p}} m_{1}^{\frac{1}{p}}+C_{1} t^{\frac{1}{q}} m_{2}^{\frac{1}{q}} .
\end{aligned}
$$

For each $k$, we set

$$
\begin{aligned}
& F_{k+1}=\left\{u \in W_{0}^{1, p}\left(\Omega, h_{1}\right): \int_{\Omega} h_{1}(x)|\nabla u|^{p} d x \geq \lambda_{k+1} \int_{\Omega} a(x)|u|^{p} d x\right\}, \\
& F_{k+1}^{\prime}=\left\{v \in W_{0}^{1, q}\left(\Omega, h_{2}\right): \int_{\Omega} h_{2}(x)|\nabla v|^{q} d x \geq \mu_{k+1} \int_{\Omega} b(x)|v|^{q} d x\right\} .
\end{aligned}
$$

For $(u, v) \in F_{k+1} \times F_{k+1}^{\prime}$, we have

$$
\begin{aligned}
I_{\lambda, \mu}(u, v) \geq\left(\frac{1}{p}-\right. & \left.\frac{\lambda}{p \lambda_{k+1}}-\frac{\epsilon}{p \lambda_{1}}\right) \int_{\Omega} h_{1}(x)|\nabla u|^{p} d x \\
& +\left(\frac{1}{q}-\frac{\mu}{q \mu_{k+1}}-\frac{\varepsilon}{q \mu_{1}}\right) \int_{\Omega} h_{2}(x)|\nabla v|^{q} d x \\
& -C_{1}\left(\int_{\Omega} h_{1}(x)|\nabla u|^{p} d x\right)^{\frac{1}{p}}-C_{1}\left(\int_{\Omega} h_{2}(x)|\nabla v|^{q} d x\right)^{\frac{1}{q}}
\end{aligned}
$$

Taking

$$
\varepsilon=\frac{1}{2} \min \left\{\frac{\lambda_{1}\left(\lambda_{k+1}-\lambda\right)}{\lambda_{k+1}}, \frac{\mu_{1}\left(\mu_{k+1}-\mu\right)}{\mu_{k+1}}, \frac{\left(\lambda-m_{1}\right) \lambda_{1}}{m_{1}}, \frac{\left(\mu-m_{2}\right) \mu_{1}}{m_{2}}\right\},
$$

we get from (3.12) and (3.13) that

$$
\beta:=\inf _{(u, v) \in F_{k+1} \times F_{k+1}^{\prime}} I_{\lambda, \mu}(u, v)
$$

and there exists $T>0$ such that

$$
\alpha:=\max _{(u, v) \in A_{1} \times A_{2}: t \geq T} I_{\lambda, \mu}\left(t^{\frac{1}{p}} u, t^{\frac{1}{q}} v\right)<\beta .
$$

Now let

$$
T A:=\left\{(t u, t v):(u, v) \in A_{1} \times A_{2}, t \geq T\right\} .
$$

Set $Q=B_{k}\left(B_{k}\right.$ represents the closed unit ball in $\left.\mathbb{R}^{k}\right), \partial Q=S^{k-1}$, and

$$
\Gamma=\left\{\gamma \in C^{0}\left(S^{k-1}, H\right): \gamma \text { is odd and } \gamma\left(S^{k-1}\right) \subset T A\right\} .
$$


For any $\gamma \in \Gamma$, by (3.14), (3.15) we obtain

$$
\gamma\left(S^{k-1}\right) \cap\left(F_{k+1} \times F_{k+1}^{\prime}\right)=\varnothing,
$$

which shows that

$$
\Gamma \subset C\left(S^{k-1}, H \backslash\left(F_{k+1} \times F_{k+1}^{\prime}\right)\right) .
$$

Let $\Gamma^{*}=\left\{\gamma \in C^{0}\left(B_{k}, H\right):\left.\gamma\right|_{S^{k-1}} \in \Gamma\right\}$. Then $\Gamma^{*}$ is nonempty.

We prove that if $\gamma \in \Gamma^{*}$ then $\gamma\left(B_{k}\right) \cap\left(F_{k+1} \times F_{k+1}^{\prime}\right) \neq \varnothing$. In fact, by the definition of $\sum_{k}, \sum_{k}^{\prime}$ there exist continuous odd surjections $\gamma_{1}: S^{k-1} \rightarrow A_{1}, \gamma_{2}: S^{k-1} \rightarrow A_{2}$. So we can define $\gamma: S^{k-1} \rightarrow A_{1} \times A_{2}$ by $\gamma=\left(\gamma_{1}, \gamma_{2}\right)$. Define $\bar{\gamma}: B_{k} \rightarrow H$ by $\bar{\gamma}(t s)=t \operatorname{T\gamma }(s)$ for any $s \in S^{k-1}$ and any $t \in[0,1]$. Thus $\bar{\gamma} \in \Gamma^{*}$. If there exists $(u, v) \in \gamma\left(B_{k}\right)$ such that

$$
\int_{\Omega} a(x)|u|^{p} d x=0, \quad \int_{\Omega} b(x)|v|^{q} d x=0
$$

then we get $\gamma\left(B_{k}\right) \cap\left(F_{k+1} \times F_{k+1}^{\prime}\right) \neq \varnothing$.

Otherwise, we consider the map $\widehat{\gamma}: S^{k} \rightarrow E$ by

$$
\widehat{\gamma}\left(x_{1}, \ldots, x_{k+1}\right)= \begin{cases}\pi \circ \gamma\left(x_{1}, \ldots, x_{k}\right), & \text { for } x_{k+1} \geq 0 \\ -\pi \circ \gamma\left(-x_{1}, \ldots,-x_{k}\right), & \text { for } x_{k+1}<0\end{cases}
$$

where

$$
\pi(u, v)=\left(\frac{u}{\int_{\Omega} a(x)|u|^{p} d x}, \frac{v}{\int_{\Omega} b(x)|v|^{q} d x}\right) .
$$

We can easily show that $\widehat{\gamma}$ is odd. Hence $\widehat{\gamma}\left(S^{k}\right)=\left(\widehat{\gamma}_{1}\left(S^{k}\right), \widehat{\gamma}_{2}\left(S^{k}\right)\right) \in \sum_{k} \times \sum_{k}^{\prime}$. On the other hand, we have,

$$
\lambda_{k+1}=\inf _{A_{1} \in \sum_{k}} \sup _{u \in A_{1}} I_{1}(u)
$$

then

$$
\lambda_{k+1} \leq \sup _{u \in \widehat{\gamma}_{1}\left(S^{k}\right)} I_{1}(u)
$$

Hence for $u \in \widehat{\gamma}_{1}\left(S^{k}\right)$, that is, for some $x \in S^{k-1}$ such that $u=\widehat{\gamma}_{1}(x)$ we have $\lambda_{k+1} \leq I_{1}(u)$. This implies that $\widehat{\gamma}_{1}(x) \in F_{k+1}$. Using the definition of $\widehat{\gamma}_{1}$, we Obtain that $\gamma_{1}(x) \in F_{k+1}$. In a similar way we Obtain that $\gamma_{2}(x) \in F_{k+1}^{\prime}$. So $\gamma\left(B_{k}\right) \cap\left\{F_{k+1} \times F_{k+1}^{\prime}\right\} \neq \varnothing$. Hence $S^{k}$ and $F_{k+1} \times F_{k+1}^{\prime}$ are $\Gamma-$ linking. The conditions of Lemma 2.6 are satisfied. So Theorem 1.2 holds for any $\lambda_{k}<\lambda<\lambda_{k+1}$, $\mu_{k}<\mu<\mu_{k+1}$ with the critical value

$$
c:=\inf _{\gamma \in \Gamma^{*}} \sup _{x \in B_{k}} I_{\lambda, \mu}(\gamma(x)) .
$$

Case 2: $\lambda=\lambda_{k}, \mu=\mu_{k}$. Let $\delta_{1} \in\left(0, \lambda_{k+1}-\lambda_{k}\right), \delta_{2} \in\left(0, \mu_{k+1}-\mu_{k}\right)$, we assume $\kappa_{n} \in\left(\lambda_{k}, \lambda_{k}+\delta_{1}\right), \tau_{n} \in\left(\mu_{k}, \mu_{k}+\delta_{2}\right)$ and $\kappa_{n} \rightarrow \lambda_{k}, \tau_{n} \rightarrow \mu_{k}$. It follows from the case $\lambda_{k}<\lambda<\lambda_{k+1}, \mu_{k}<\mu<\mu_{k+1}$ that there exists $c_{n}$ to be the critical value of $I_{\kappa_{n}}, \tau_{n}$ and $\left(u_{n}, v_{n}\right)$ be the critical point corresponding to $c_{n}$ satisfying 
$I_{\kappa_{n}, \tau_{n}}^{\prime}\left(u_{n}, v_{n}\right)=0$ for any $n$. Thus, for any $\left(\tau_{n}, \kappa_{n}\right)$ there exists a corresponding set $\Gamma_{n}^{*}$ such that the critical value $c_{n}$ is characterized by

$$
c_{n}:=\inf _{\gamma \in \Gamma_{n}^{*}} \sup _{x \in B_{k}} I_{\mathcal{K}_{n}, \tau_{n}}(\gamma(x))
$$

For $(u, v) \in F_{k+1} \times F_{k+1}^{\prime}$, let

$$
\varepsilon=\min \left\{\frac{\left(\lambda_{k+1}-\lambda_{k}-\delta_{1}\right) \lambda_{1}}{2 \lambda_{k+1}}, \frac{\left(\mu_{k+1}-\mu_{k}-\delta_{2}\right) \mu_{1}}{2 \mu_{k+1}}\right\},
$$

we obtain

$$
\begin{aligned}
I_{\kappa_{n}, \tau_{n}}(u, v) \geq \frac{1}{p} & \left(1-\frac{\kappa_{n}}{\lambda_{k+1}}-\frac{\varepsilon}{\lambda_{1}}\right)\|u\|_{h_{1}, p}^{p}+\frac{1}{q}\left(1-\frac{\tau_{n}}{\mu_{k+1}}-\frac{\varepsilon}{\mu_{1}}\right)\|v\|_{h_{2}, q}^{q} \\
& -C_{1}\left(\|u\|_{h_{1}, p}+\|v\|_{h_{2}, q}\right) \\
\geq \frac{1}{p} & \left(1-\frac{\lambda_{k}+\delta_{1}}{\lambda_{k+1}}-\frac{\varepsilon}{\lambda_{1}}\right)\|u\|_{h_{1}, p}^{p}+\frac{1}{q}\left(1-\frac{\mu_{k}+\delta_{2}}{\mu_{k+1}}-\frac{\varepsilon}{\mu_{1}}\right)\|v\|_{h_{2}, q}^{q} \\
& -C_{1}\left(\|u\|_{h_{1}, p}+\|v\|_{h_{2}, q}\right),
\end{aligned}
$$

which implies that there exists $\beta_{0}>0$ such that

$$
\begin{aligned}
\sup _{x \in B_{k}} I_{\kappa_{n}, \tau_{n}}(\gamma(x)) & \geq \sup _{(u, v) \in \gamma\left(B_{k}\right) \cap\left(F_{k+1} \times F_{k+1}^{\prime}\right)} I_{\kappa_{n}, \tau_{n}}(\gamma(x)) \\
& \geq \inf _{(u, v) \in F_{k+1} \times F_{k+1}^{\prime}} I_{\mathcal{K}_{n}, \tau_{n}}(\gamma(x)) \\
& \geq \beta_{0}
\end{aligned}
$$

for all $\gamma \in \Gamma_{n}^{*}$. Then one has $c_{n} \geq \beta_{0}$, we get that there exists a subsequence of critical points $\left(u_{n}, v_{n}\right)$ which converges to the desired critical point of $I_{\lambda_{k}, u_{k}}$.

\section{Acknowledgments}

The authors would like to thank the referees for their suggestions and helpful comments which improved the presentation of the original manuscript. The second author was supported by Vietnam National Foundation for Science and Technology Development (NAFOSTED).

\section{References}

[1] G.A. Afrouzi, S. Mahdavi ANd Z. NAGHizAdeH, Existence of multiple solutions for a class of $(p, q)$-Laplacian systems, Nonlinear Anal., 72 (2010), 2243-2250.

[2] G.A. Afrouzi, S. Mahdavi And N.B. Zographopoulos, Existence of solutions for non-uniformly nonlinear elliptic systems, Electron. J. Diff. Equ., 167 (2011), 1-9. 
[3] A. Ambrosetti AND P. H. Rabinowitz, Dual variational methods in critical point theory and applications, J. Funct. Anal., 14 (1973), 349-381.

[4] P. Bartolo, V. Benci And D. Fortunato, Abstract critical point theorems and application to some nonlinear problems with strong resonance at infinity, Nonlinear Anal., 7 (1983), 981-1012.

[5] P. CAldiroli And R. Musina, On a variational degenerate elliptic problem, Nonlinear Differ. Eqns. Appl. (NoDEA), 7 (2000), 189-199.

[6] N.T. CHUNG, Existence of weak solutions for a nonuniformly elliptic nonlinear system in $\mathbb{R}^{N}$, Electron. J. Diff. Equ., 119 (2008), 1-10.

[7] N.T. CHUNG, On the existence of weak solutions for a degenerate and singular elliptic systems in $\mathbb{R}^{N}$, Acta. Appl. Math., 110 (2010), 47-56.

[8] N.T. CHUNG, Existence of infinitely many solutions for degenerate and singular elliptic systems with indefinite concave nonlinearities, Electron. J. Diff. Equ., 30 (2011), 1-12.

[9] N. T. CHUNG AND H. Q. TOAN, On a class of degenerate and singular elliptic systems in bounded domains, J. Math. Anal. Appl., 360 (2009) 422-431.

[10] M. CUESTA AND H.R. QUOIRIN, A weighted eigenvalue problem for the p-Laplacian plus a potential, Nonlinear Differential Equations and Applications (NoDEA), 16 (2009), 469-491.

[11] P. DrÁBEK, A. KUfNer AND F. NiClosi, Quasilinear elliptic equations with degenerate and singularities, Vol. 5 of de Gruyter Series in Nonlinear Analysis and Applications, Walter and Gruyter and Co. Berlin, 1997.

[12] P. DrÁbeK AND J. Milota, Methods of Nonlinear Analysis, Applications to Differential Equations, Birkhäuser Verlag AG, Basel-Boston-Berlin, 2007.

[13] A. LÊ AND K. SCHMITT, Variational eigenvalues of degenerate eigenvalue problem for weighted p-Laplacian, Advanced Nonlinear Studies, 5(2005), 553-565.

[14] O.H. MiYagaKi AND R.S. RODRIGUES, On positive solution for a class of degenerate quasilinear elliptic positone / semipositone systems, Nonlinear Anal., 70 (2009), 99-116.

[15] Z.Q. OU AND C.L. TANG, Existence and multiplicity results for some elliptic system at resonance, Nonlinear Anal., 71 (2009), 2660-2666.

[16] S. Z. SONG AND C. L. TANG, Existence theorem for quasilinear elliptic resonance problems, J. Southwest China Normal Univ. (Natural science), 30 (1) (2005), 1-6.

[17] H. Q. TOAN AND N. T. CHUNG, Existence of weak solution for a class of nonuniformly nonlinear elliptic equation in unbounded domains, Nonlinear Anal., 70 (2009), 3987-3996. 
[18] J. ZHANG AND Z. ZHANG, Existence results for some nonlinear elliptic systems, Nonlinear Anal., 71 (2009), 2840-2846.

[19] G. ZHANG AND Y. WANG, Some existence results for a class of degenerate semilinear elliptic systems, J. Math. Anal. Appl., 333 (2007), 904 - 918.

[20] X. X. ZHAO AND C. L. TANG, Resonance problems for $(p, q)$-Laplacian systems, Nonlinear Anal., 72 (2010), 1019-1030.

[21] N.B. Zographopoulos, On a class of degenerate potential elliptic systems, Nonlinear Differ. Equ. Appl., 11 (2004), 191-199.

[22] N.B. ZOGRAPHOPOUlOS, On the principal eigenvalue of degenerate quasilinear elliptic systems, Math. Nachr., 281(9) (2008), 1351-1365.

Department of Mathematics, Faculty of Mathematical Sciences,

University of Mazandaran, Babolsar, Iran

email:afrouzi@umz.ac.ir, smahdavi@umz.ac.ir

Department of Science Management \& International Cooperation,

Quang Binh University, 312 Ly Thuong Kiet,

Dong Hoi, Quang Binh, Vietnam

email:ntchung82@yahoo.com 